\title{
Modus Ponens Tollens for RU-Implications
}

\author{
Isabel Aguiló ${ }^{1,2}$ (D), Sebastia Massanet ${ }^{1,2}$ (D), Juan Vicente Riera ${ }^{1,2}$ (D), \\ and Daniel Ruiz-Aguilera ${ }^{1,2(\bowtie)}$ (D) \\ 1 Department of Mathematics and Computer Science, \\ University of the Balearic Islands, 07122 Palma, Spain \\ \{isabel.aguilo, s.massanet, jvicente.riera, daniel.ruiz\}@uib.es \\ ${ }^{2}$ Health Research Institute of the Balearic Islands (IdISBa), 07010 Palma, Spain
}

\begin{abstract}
In fuzzy rules based systems, fuzzy implication functions are usually considered to model fuzzy conditionals and to perform forward and backward inferences. These processes are guaranteed by the fulfilment of the Modus Ponens and Modus Tollens properties by the fuzzy implication function with respect to the considered conjunction and fuzzy negation. In this paper, we investigate which residual implications derived from uninorms satisfy both Modus Ponens and Modus Tollens properties with respect to the same t-norm and a fuzzy negation simultaneously. The most usual classes of uninorms are considered and many solutions are obtained which allow to model the fuzzy conditionals in a fuzzy rules based systems (and perform backward and forward inferences) with a unique residual implication derived from a uninorm.
\end{abstract}

Keywords: Fuzzy implication function $\cdot$ Modus Ponens $\cdot$ Modus

Tollens · Uninorm

\section{Introduction}

Fuzzy implication functions have been extensively studied in the last decades (see $[3,4,18]$ and references therein). There exist two main reasons to support the great effort made by the scientific community in this field. First, fuzzy implication functions have proved useful in many applications ranging from approximate reasoning to image processing, including fuzzy control, fuzzy relational equations, fuzzy DI-subsethood measures or computing with words, among other fields. The second reason is a direct consequence of their definition, which imposes only some monotonicities and corner conditions to ensure that they generalize the binary implication when restricted to $\{0,1\}$. This fact opens a plethora of additional properties which, although they are studied from a theoretical point of view, are useful to obtain feasible and more adequate fuzzy implication functions in the applications.

Two of such additional properties are the (generalized) Modus Ponens and Modus Tollens. These properties are of paramount importance in approximate

(C) Springer Nature Switzerland AG 2020

M.-J. Lesot et al. (Eds.): IPMU 2020, CCIS 1238, pp. 788-801, 2020.

https://doi.org/10.1007/978-3-030-50143-3_61 
reasoning. Indeed, in any fuzzy rules based system, the fuzzy conditionals are usually modelled by fuzzy implication functions. However, in order to perform backward and forward inferences, the considered fuzzy implication functions must satisfy the aforementioned (generalized) Modus Ponens and Modus Tollens properties with respect to the conjunction and fuzzy negation considered in the system. These properties are usually carried out through the Compositional Rule of Inference (CRI) of Zadeh, based on the sup $-T$ composition, where $T$ is a tnorm (see for instance [5] or Chapter 7 in [3]). Applying this approach, the (generalized) Modus Ponens and Modus Tollens are usually expressed by the following two functional inequalities:

$$
\begin{gathered}
T(x, I(x, y)) \leq y, \text { for all } x, y \in[0,1], \\
T(N(y), I(x, y)) \leq N(x), \text { for all } x, y \in[0,1],
\end{gathered}
$$

where $T$ is a t-norm, $I$ a fuzzy implication function and $N$ a fuzzy negation. These properties have been studied in the literature for the most usual families of fuzzy implication functions such as $(S, N), R, Q L$ and $D$-implications derived from t-norms and t-conorms $[2,3,16,18,23-25]$ or from uninorms $[14,15]$. Even recently, a whole new line of research has been proposed in which the t-norm $T$ is generalized to a more general conjunction such as a conjunctive uninorm [19] or an overlap function [8], leading to the so-called $U$-Modus Ponens or $O$-Modus Ponens.

Although the functional inequalities of Modus Ponens and Modus Tollens have quite similar expressions, it is well-known that both properties are not equivalent. Thus, in $[23,24]$, the simultaneous fulfillment of both properties was studied for the first time for some restricted classes of $(S, N), R, Q L$ and $D$ implications. It was proved that when the fuzzy negation $N$ is a strong negation, both properties are equivalent if the fuzzy implication function satisfies the contrapositive symmetry with respect to $N$. The importance of the disposal of fuzzy implication functions satisfying both properties lies on the possibility of considering a unique implication to model fuzzy conditionals regardless of whether backward or forward inference processes have to be performed.

Following this line of research, in this paper, we analyze which residual implications derived from uninorms, or $R U$-implications for short, satisfy both the Modus Ponens and the Modus Tollens properties with respect to the same tnorm $T$ and a fuzzy negation $N$ (continuous, but not necessarily strong). It has to be said that the Modus Ponens property had been already studied for this family of uninorms in [15] and the Modus Tollens property was analyzed in [14]. However, while in [15], the results were given in terms of the t-norm $T_{U}$ associated to the uninorm $U$, in [14] the results were presented for each class of uninorms separately. This fact makes it difficult to coordinate the results of both studies in order to find $R U$-implications satisfying both properties. This is the main goal of this paper in addition to find some cases for which the fulfillment of one property implies the fulfillment of the other one.

The paper is organized as follows. In the next section we recall some basic definitions and properties on fuzzy implication functions and uninorms. In Sect. 3, 
we introduce the so-called Modus Ponens Tollens property and we discuss its fulfillment when the fuzzy implication function satisfies the contrapositive symmetry with respect to a strong negation. After that, in Sect. 4, the Modus Ponens Tollens property is studied in depth for $R U$-implications depending on the class of the uninorm $U$ considered. The paper ends with some conclusions and future work.

\section{Preliminaries}

We will suppose the reader to be familiar with the theory of t-norms, t-conorms and fuzzy negations (all necessary results and notations can be found in [11]). We also suppose that some basic facts on uninorms are known (see for instance [9]) as well as their most usual classes (see [13] for a complete survey), that is, uninorms in $\mathcal{U}_{\min }([9])$, representable uninorms $([9])$, idempotent uninorms $([6,12,22])$ and uninorms continuous in the open unit square $([10])$.

We recall here only some facts on implications and uninorms in order to establish the necessary notation that we will use along the paper.

Definition 1 ([3]). A binary operator $I:[0,1] \times[0,1] \rightarrow[0,1]$ is said to be a fuzzy implication function, or a fuzzy implication, if it satisfies:

(I1) $I(x, z) \geq I(y, z)$ when $x \leq y$, for all $z \in[0,1]$.

(I2) $I(x, y) \leq I(x, z)$ when $y \leq z$, for all $x \in[0,1]$.

(I3) $I(0,0)=I(1,1)=1$ and $I(1,0)=0$.

Note that, from the definition, it follows that $I(0, x)=1$ and $I(x, 1)=1$ for all $x \in[0,1]$ whereas the symmetrical values $I(x, 0)$ and $I(1, x)$ are not derived from it.

Definition 2 ([3]). A function $N:[0,1] \rightarrow[0,1]$ is called a fuzzy negation if it is decreasing, $N(0)=1$ and $N(1)=0$. If $N$ is a fuzzy negation that is strictly decreasing and continuous, it will be called strict, and if it is involutive, $N(N(x))=x$ for all $x \in[0,1]$, then it will be called strong.

Definition 3 ([3]). Let $T$ be a t-norm. A function $N_{T}:[0,1] \rightarrow[0,1]$ defined as

$$
N_{T}(x)=\sup \{y \in[0,1] \mid T(x, y)=0\}, x \in[0,1]
$$

is called the natural negation of $T$ or the negation induced by $T$.

Definition 4 ([9]). A uninorm is a two-place function $U:[0,1]^{2} \rightarrow[0,1]$ which is associative, commutative, increasing in each place and such that there exists some element $e \in[0,1]$, called neutral element, such that $U(e, x)=x$ for all $x \in[0,1]$.

Evidently, a uninorm with neutral element $e=1$ is a t-norm and a uninorm with neutral element $e=0$ is a t-conorm. For any other value $e \in] 0,1[$ the operation works as a t-norm in the $[0, e]^{2}$ square, as a t-conorm in $[e, 1]^{2}$ and 
its values are between the minimum and the maximum in the set of points $A(e)$ given by

$$
A(e)=[0, e[\times] e, 1] \cup] e, 1] \times[0, e[.
$$

We will usually denote a uninorm with neutral element $e$ and underlying t-norm $T_{U}$ and t-conorm $S_{U}$ by $U \equiv\left\langle T_{U}, e, S_{U}\right\rangle$. For any uninorm it is satisfied that $U(0,1) \in\{0,1\}$ and a uninorm $U$ is called conjunctive if $U(1,0)=0$ and disjunctive when $U(1,0)=1$. On the other hand, let us recall the most usual classes of uninorms in the literature that will be used along the paper. We start with the class of uninorms in $\mathcal{U}_{\text {min }}$.

Definition 5. Let $U$ be a conjunctive uninorm with neutral element $e \in] 0,1[$. If the mapping $x \mapsto U(x, 1)$ is continuous except in $x=e$, then it is said that $U$ is a uninorm in $\mathcal{U}_{\min }$.

Theorem 1 ([9]). Let $U:[0,1]^{2} \rightarrow[0,1]$ be a function. Then $U$ is a uninorm in $\mathcal{U}_{\min }$ if and only if $U$ is given by

$$
U(x, y)= \begin{cases}e T_{U}\left(\frac{x}{e}, \frac{y}{e}\right) & \text { if }(x, y) \in[0, e]^{2}, \\ e+(1-e) S_{U}\left(\frac{x-e}{1-e}, \frac{y-e}{1-e}\right) & \text { if }(x, y) \in[e, 1]^{2}, \\ \min (x, y) & \text { if }(x, y) \in A(e)\end{cases}
$$

where $T_{U}$ is a t-norm, and $S_{U}$ is a t-conorm. We will denote a uninorm in $\mathcal{U}_{\text {min }}$ with underlying $t$-norm $T_{U}$, underlying t-conorm $S_{U}$ and neutral element $e$ as $U \equiv\left\langle T_{U}, e, S_{U}\right\rangle_{\min }$

The class of idempotent uninorms, that satisfy $U(x, x)=x$ for all $x \in[0,1]$, was characterized first in [6] for those uninorms with a lateral continuity and in [12] for the general case. An improvement of this last result was done in [22] as follows.

Theorem 2 ([22]). $U$ is an idempotent uninorm with neutral element $e \in[0,1]$ if and only if there exists a non increasing function $g:[0,1] \rightarrow[0,1]$, symmetric with respect to the identity function, with $g(e)=e$, such that

$$
U(x, y)= \begin{cases}\min (x, y) & \text { if } y<g(x) \text { or }\left(y=g(x) \text { and } x<g^{2}(x)\right) \\ \max (x, y) & \text { if } y>g(x) \text { or }\left(y=g(x) \text { and } x>g^{2}(x)\right) \\ x \text { or } y & \text { if } y=g(x) \text { and } x=g^{2}(x)\end{cases}
$$

being commutative in the points $(x, y)$ such that $y=g(x)$ with $x=g^{2}(x)$.

Any idempotent uninorm $U$ with neutral element $e$ and associated function $g$ will be denoted by $U \equiv\langle g, e\rangle_{\text {ide }}$ and the class of idempotent uninorms will be denoted by $\mathcal{U}_{\text {ide }}$. Obviously, for any of these uninorms, the underlying t-norm is the minimum and the underlying t-conorm is the maximum. 
Definition 6 ([9]). Let e be in $] 0,1\left[\right.$. A binary operation $U:[0,1]^{2} \rightarrow[0,1]$ is a representable uninorm if and only if there exists a strictly increasing function $h:[0,1] \rightarrow[-\infty,+\infty]$ with $h(0)=-\infty, h(e)=0$ and $h(1)=+\infty$ such that

$$
U(x, y)=h^{-1}(h(x)+h(y))
$$

for all $(x, y) \in[0,1]^{2} \backslash\{(0,1),(1,0)\}$ and $U(0,1)=U(1,0) \in\{0,1\}$. The function $h$ is usually called an additive generator of $U$.

Any representable uninorm $U$ with neutral element $e$ and additive generator $h$ will be denoted by $U \equiv\langle h, e\rangle_{\text {rep }}$ and the class of representable uninorms will be denoted by $\mathcal{U}_{\text {rep }}$. For any of these uninorms the underlying t-norm and tconorm are always strict. For all representable uninorm $U$, a strong negation can be defined from $U$ as $N_{U}(x)=h^{-1}(-h(x))$ for all $x \in[0,1]$.

Another studied class of uninorms is $\mathcal{U}_{\text {cos }}$, composed by all uninorms continuous in $] 0,1\left[^{2}\right.$. They were introduced and characterized in [10] as follows.

Theorem 3 ([10]). Suppose $U$ is a uninorm continuous in $] 0,1\left[^{2}\right.$ with neutral element $e \in] 0,1[$. Then one of the following cases is satisfied:

(a) There exist $u \in\left[0, e\left[, \lambda \in[0, u]\right.\right.$, two continuous t-norms $T_{1}$ and $T_{2}$ and $a$ representable uninorm $R$ such that $U$ can be represented as

$$
U(x, y)= \begin{cases}\lambda T_{1}\left(\frac{x}{\lambda}, \frac{y}{\lambda}\right) & \text { if }(x, y) \in[0, \lambda]^{2}, \\ \lambda+(u-\lambda) T_{2}\left(\frac{x-\lambda}{u-\lambda}, \frac{y-\lambda}{u-\lambda}\right) & \text { if }(x, y) \in[\lambda, u]^{2}, \\ u+(1-u) R\left(\frac{x-\lambda}{1-\lambda}, \frac{y-\lambda}{1-\lambda}\right) & \text { if }(x, y) \in[\lambda, u]^{2}, \\ 1 & \text { if } \min (x, y) \in] \lambda, 1] \\ & \text { and } \max (x, y)=1, \\ \lambda \operatorname{or} 1 & \text { if }(x, y) \in\{(\lambda, 1),(1, \lambda)\}, \\ \min (x, y) & \text { elsewhere. }\end{cases}
$$

(b) There exist $v \in] e, 1] \omega \in[v, 1]$, two continuous t-conorms $S_{1}$ and $S_{2}$ and $a$ representable uninorm $R$ such that $U$ can be represented as

$$
U(x, y)= \begin{cases}v R\left(\frac{x}{v}, \frac{y}{v}\right) & \text { if }(x, y) \in] 0, v{ }^{2}, \\ v+(\omega-v) S_{1}\left(\frac{x-v}{\omega-v}, \frac{y-v}{\omega-v}\right) & \text { if }(x, y) \in[v, \omega]^{2}, \\ \omega+(1-\omega) S_{2}\left(\frac{x-\omega}{1-\omega}, \frac{y-\omega}{1-\omega}\right) & \text { if }(x, y) \in[\omega, 1]^{2}, \\ 0 & \text { if } \max (x, y) \in[0, \omega[ \\ & \text { and } \min (x, y)=0, \\ \omega \operatorname{or} 0 & \text { if }(x, y) \in\{(0, \omega),(\omega, 0)\}, \\ \max (x, y) & \text { elsewhere. }\end{cases}
$$

Now we will recall residual implications from uninorms: $R U$-implications.

Definition 7 ([7]). Let $U$ be a uninorm. The residual operation derived from $U$ is the binary operation given by

$$
I_{U}(x, y)=\sup \{z \in[0,1] \mid U(x, z) \leq y\} \text { for all } x, y \in[0,1] .
$$


Proposition 1 ([7]). Let $U$ be a uninorm and $I_{U}$ its residual operation. Then $I_{U}$ is a fuzzy implication if and only if the following condition holds

$$
U(x, 0)=0 \text { for all } x<1 .
$$

In this case $I_{U}$ is called an $R U$-implication.

This includes all conjunctive uninorms but also many disjunctive ones, for instance in the classes of representable uninorms (see [7]) and idempotent uninorms (see [20]).

Some properties of $R U$-implications have been studied involving the main classes of uninorms, those previously stated: uninorms in $\mathcal{U}_{\min }$, idempotent uninorms and representable uninorms (for more details see $[1,3,7,17,20,21]$ ).

\section{Modus Ponens Tollens}

First of all, let us recall the definition of the Modus Ponens and the Modus Tollens in the framework of fuzzy logic.

Definition 8. Let $I$ be a fuzzy implication function and $T$ a $t$-norm. It is said that $I$ satisfies the Modus Ponens property with respect to $T$ if

$$
T(x, I(x, y)) \leq y \text { for all } x, y \in[0,1]
$$

Definition 9. Let I be a fuzzy implication function, $T$ a t-norm and $N$ a fuzzy negation. It is said that $I$ satisfies the Modus Tollens property with respect to $T$ and $N$ if

$$
T(N(y), I(x, y)) \leq N(x) \text { for all } x, y \in[0,1] .
$$

The Modus Ponens and Modus Tollens properties have been studied for different types of implications, usuallly taking into account continuous t-norms $T$ and continuous fuzzy negations $N$. If we consider $R U$-implications, (MP) and (MT) have been studied in depth in [15] and [14], respectively. These properties are not equivalent in general, as it is stated in the following examples. First, we have a fuzzy implication function that satisfies (MP) but not (MT).

Example 1. Consider $U \equiv\left\langle h, \frac{3}{4}\right\rangle_{\text {rep }}$ a representable uninorm with $T_{U}=T_{\mathbf{P}}$, the product t-norm (with additive generator $t_{U}(x)=-\ln (x)$ up to a multiplicative constant) and $S_{U}$ any strict t-conorm. Let us consider its residual implication $I_{U}$ which will be given later in Proposition 10. Let us also consider $T=T_{\mathbf{P}}$ and the negation $N(x)=\frac{1-x}{1+10 x}$ which belongs to the family of Sugeno negations with $\lambda=10$. In this case, $I_{U}$ safisfies (MP) with respect to $T$ by using Proposition 9 in [15]. However, $I_{U}$ does not satisfy (MT) with respect to $T$ and $N$ (just taking $x=0.7$ and $y=0.5$ in Eq. (MP)).

Next example provides an $R U$-implication that satisfies (MT) but not (MP). 
Example 2. Let $U \equiv\left\langle h, \frac{1}{2}\right\rangle_{\text {rep }}$ be a representable uninorm with additive generator $h(x)=\ln \left(\frac{x}{1-x}\right)$ for all $x \in[0,1]$. Let $T$ be a t-norm whose expression is given by the ordinal sum $T \equiv\left(\left\langle 0, \frac{1}{2}, T_{\mathbf{P}}\right\rangle,\left\langle\frac{1}{2}, 1, T_{1}\right\rangle\right)$ with $T_{1}$ any continuous t-norm and let us consider the continuous fuzzy negation $N$ given by

$$
N(x)= \begin{cases}1-x & \text { if } x \leq \frac{1}{2} \\ \sqrt{x-x^{2}} & \text { otherwise }\end{cases}
$$

In this case, $I_{U}$ is given by

$$
I_{U}(x, y)= \begin{cases}1 & \text { if }(x, y) \in\{(0,0),(1,1)\} \\ \frac{(1-x) y}{x+y-2 x y} & \text { otherwise }\end{cases}
$$

According to Proposition 5.3.20-(ii) in [14], $I_{U}$ satisfies (MT) with respect to $T$ and $N$. However, a simple computation shows that $g:[0,1] \rightarrow[0,1]$ given by $g(x)=-\ln \left(\frac{2}{1+e^{x}}\right)$ is not subadditive (for instance, take $x=0.3$ and $y=0.2$ ) and therefore, by using Proposition 10 in [19], $I_{U}$ does not satisfy (MP) with respect to $T$.

Then, as we have seen, (MT) and (MP) are not equivalent in general, and the question about which fuzzy implication functions satisfy both properties with respect to the same t-norm $T$ and fuzzy negation $N$ is worthy to study.

Definition 10. Let $I$ be a fuzzy implication function, $T$ a t-norm and $N$ a fuzzy negation. It is said that I satisfies the Modus Ponens Tollens (MPT) property with respect to $T$ and $N$ whenever Eqs. (MP) and (MT) are satisfied simultaneously.

Remark 1. Note that, when $x \leq y$ we have $N(y) \leq N(x)$ and then (MT) trivially holds in these cases. Similarly, (MP) is satisfied in these cases. Thus, both properties need to be checked only in points $(x, y) \in[0,1]^{2}$ where $y<x$.

Anyway, a special case that can be considered is when $I$ satisfies the contrapositive symmetry with respect to $N$. Contrapositive symmetry is a well known property, which is related to the Modus Ponens Tollens as it is stated in the following results.

Definition 11. Consider I a fuzzy implication function and $N$ a fuzzy negation. Then I satisfies the contrapositive symmetry with respect to $N$ if

$$
I(x, y)=I(N(y), N(x)) \text { for all } x, y \in[0,1] .
$$

Theorem 4 ([24]). Consider I a fuzzy implication function, $T$ a t-norm and $N$ a strong negation. If I satisfies the contrapositive symmetry with respect to $N$, then I satisfies (MP) with respect to $T$ if and only if I satisfies (MT) with respect to $N$ and $T$. 
From the result above, in the case that a fuzzy implication function $I$ satisfies (CP) with respect to $N$, only one of (MP) or (MT) needs to be checked in order to satisfy (MPT). Now, let us recall the result on contrapositive symmetry for residual implications derived from idempotent uninorms.

Proposition 2 ([20]). Consider $U \equiv\langle g, e\rangle_{\text {ide }}$ an idempotent uninorm with $g(0)=1, I_{U}$ its residual implication and $N$ a strong negation. Then $I_{U}$ satisfies (CP) with respect to $N$ if and only if $g=N$.

As a consequence of the previous result, we have infinite $R U$-implications that satisfy (MPT) for any t-norm T, by using Proposition 5.3.14 in [14].

Corollary 1. Let $N$ be a strong negation, $U \equiv\langle N, e\rangle_{\text {ide }}$ an idempotent uninorm, $I_{U}$ its residual implication, and $T$ a t-norm. Then $I_{U}$ satisfies (MPT) with respect to $T$ and $N$.

Coming up next, we recall the case of (CP) for uninorms continuous in $] 0,1\left[^{2}\right.$.

Proposition 3 ([21]). Let $U$ be a uninorm in $\mathcal{U}_{\cos }$ such that $U(0, x)=0$ for all $x<1, I_{U}$ its residual implication and $N$ a strong negation. Then $I_{U}$ satisfies (CP) with respect to $N$ if and only if $U$ is representable and $N=N_{U}$.

\section{Modus Ponens Tollens for Implications Derived from Different Classes of Uninorms}

In this section we investigate the Modus Ponens Tollens property (MPT) for fuzzy implication functions derived from three well known classes of uninorms.

\subsection{Case When $U$ is a Uninorm in $\mathcal{U}_{\text {min }}$}

In this section we will deal with $R U$-implications derived from uninorms in $\mathcal{U}_{\text {min }}$, that is, uninorms $U \equiv\left\langle T_{U}, e, S_{U}\right\rangle_{\min }$ with neutral element $\left.e \in\right] 0,1[$. Recall that for this kind of uninorms, $R U$-implications have the following structure.

Proposition 4 (Theorem 5.4.7 in [3]). Let $U \equiv\left\langle T_{U}, e, S_{U}\right\rangle_{\min }$ a uninorm in $\mathcal{U}_{\min }$ and $I_{U}$ its residual implication. Then

$$
I_{U}(x, y)= \begin{cases}1 & \text { if } x \leq y<e \\ e I_{T_{U}}\left(\frac{x}{e}, \frac{y}{e}\right) & \text { if } y<x \leq e \\ e+(1-e) I_{S_{U}}\left(\frac{x-e}{1-e}, \frac{y-e}{1-e}\right) & \text { if } e \leq x \leq y \\ e & \text { if } e \leq y<x \\ y & \text { elsewhere. }\end{cases}
$$

For this family of $R U$-implications we have the following result.

Proposition 5. Let $U \equiv\left\langle T_{U}, e, S_{U}\right\rangle_{\min }$ a uninorm in $\mathcal{U}_{\min }$ and $I_{U}$ its residual implication. Let $T$ be a continuous $t$-norm, and $N$ be a continuous fuzzy negation with fixed point $s \in] 0,1[$. Then, it holds that: 
- If $I_{U}$ satisfies (MPT) with $T$ and $N$, then $T$ is nilpotent with normalized additive generator $t:[0,1] \rightarrow[0,1]$ and associated negation $N_{T}(x)=t^{-1}(1-$ $t(x))$ for all $x \in[0,1]$ such that $N(y) \leq N_{T}(y)$ for all $y \leq e$.

Thus, from now on, let us consider $T$ satisfying the previous conditions. In this case,

(i) If $T_{U}=\min$ then $I_{U}$ always satisfies (MPT) with respect to $T$ and $N$.

(ii) If $T_{U}$ is a strict t-norm with additive generator $t_{U}$ and either $s \geq e$ or $N(y)=N_{T}(y)$ for all $y \leq e$, then $I_{U}$ satisfies (MPT) with respect to $T$ and $N$ if and only if the following condition holds:

$\left(\star_{1}\right)$ Function $g:[0, t(0)] \rightarrow[t(e), 1]$ given by the formula $g(x)=t\left(e_{U}^{-1}(x)\right)$ is subadditive.

(iii) If $T_{U}$ is a strict $t$-norm with additive generator $t_{U}, s<e$ and $N(y)<N_{T}(y)$ for some $y \leq e$, then $I_{U}$ satisfies (MPT) with respect to $T$ and $N$ if and only if Property $\left(\star_{1}\right)$ is fulfilled and the following condition holds:

$\left(\star_{2}\right)$ For all $y<x<e$,

$$
e t_{U}^{-1}\left(t_{U}\left(\frac{y}{e}\right)-t_{U}\left(\frac{x}{e}\right)\right) \leq t^{-1}(t(N(x))-t(N(y))) .
$$

(iv) If $T_{U}$ is a nilpotent $t$-norm with additive generator $t_{U}$ and either $s \geq e$ or $N=N_{T}$, then $I_{U}$ satisfies (MPT) with respect to $T$ and $N$ if and only if Property $\left(\star_{1}\right)$ and the following property holds:

$\left(\star_{3}\right)$ For all $x \leq e$

$$
e \cdot N_{T_{U}}\left(\frac{x}{e}\right) \leq N_{T}(x)
$$

(v) If $T_{U}$ is a nilpotent $t$-norm with additive generator $t_{U}, s<e$ and $N(y)<$ $N_{T}(y)$ for some $y \leq e$, then $I_{U}$ satisfies (MPT) with respect to $T$ and $N$ if and only if Properties $\left(\star_{1}\right),\left(\star_{2}\right)$ and $\left(\star_{3}\right)$ hold.

Example 3. Let us consider the uninorm $U \equiv\left\langle T_{\mathbf{M}}, e, S_{U}\right\rangle_{\min }$ with $T_{\mathbf{M}}$ is the minimum t-norm and $S_{U}$ any t-conorm. Let $T_{\mathbf{L}}$ be the Lukasiewicz t-norm and $N=N_{c}$ the classical negation given by $N_{c}(x)=1-x$ for all $x \in[0,1]$. Thus, from the previous proposition, taking into account that $N_{c}(x)=1-x \leq N_{T_{\mathbf{L}}}(x)=$ $1-x, I_{U}$ satisfies $(\mathbf{M P T})$ with respect to $T_{\mathbf{L}}$ and $N_{c}$.

Example 4. Let us take now $U \equiv\left\langle T_{\mathbf{P}}, \frac{1}{2}, S_{U}\right\rangle_{\min }$ with $S_{U}$ any t-conorm. Let $T_{\mathbf{L}}$ be the Eukasiewicz t-norm (with additive generator $t(x)=1-x$ up to a multiplicative constant), and $N=N_{c}$. In this example, we are under the conditions of Case (ii) from the previous result, and it remains only to prove that $g:[0,1] \rightarrow\left[\frac{1}{2}, 1\right]$ given by $g(x)=1-\frac{1}{2} e^{-x}$ is subadditive. A straightforward computation ensures this fact. Therefore, $I_{U}$ satisfies (MPT) with respect to $T_{\mathbf{L}}$ and $N_{c}$.

Example 5. Let us consider $U \equiv\left\langle T_{\mathbf{P}}, \frac{1}{2}, S_{U}\right\rangle_{\min }$ with $S_{U}$ any t-conorm. Let $T=$ $T_{\mathrm{L}}$ and let us take

$$
N(x)= \begin{cases}1-2 x & \text { if } x \leq \frac{1}{2} \\ 0 & \text { elsewhere }\end{cases}
$$


a continuous fuzzy negation with fixed point $s=\frac{1}{3}$. Note that in this case $N_{T}=N_{c}(x)=1-x$ and $N\left(\frac{1}{4}\right)<N_{T}\left(\frac{1}{4}\right)$. Thereby, we are under the conditions of Case $\left(\right.$ iii) of the previous proposition, and then Properties $\left(\star_{1}\right)$ and $\left(\star_{2}\right)$ must be checked. A simple computation shows that $g:[0,1] \rightarrow\left[\frac{1}{2}, 1\right]$ given by $g(x)=1-\frac{1}{2} e^{-x}$ is subadditive and so Property $\left(\star_{1}\right)$ is fulfilled. With respect to Property $\left(\star_{2}\right)$, we obtain the inequality $\frac{y}{2 x} \leq 1-2(x-y)$ which is valid for $y<x<\frac{1}{2}$. Consequently, $I_{U}$ satisfies (MPT) with respect to $T_{\mathbf{L}}$ and $N$.

Example 6. Let us consider $U \equiv\left\langle T_{\mathbf{L}}, \frac{1}{2}, S_{U}\right\rangle_{\min }$ with $S_{U}$ any t-conorm. Let us consider $T=T_{\mathbf{L}}$ and the same negation $N$ used in the previous example. In this case, again it holds that $N_{T}=N_{c}$ and it follows that $N(x)<N_{T}(x)$ (take for instance $\left.x=\frac{1}{3}, N\left(\frac{1}{3}\right)=\frac{1}{3}<N_{T_{\mathbf{L}}}\left(\frac{1}{3}\right)=\frac{2}{3}\right)$. Now, we are under the conditions of Case (v) from the previous result, and then we must check Properties $\left(\star_{1}\right)$, $\left(\star_{2}\right)$ and $\left(\star_{3}\right)$. Property $\left(\star_{1}\right)$ follows directly. With respect to Property $\left(\star_{2}\right)$, we have $g:[0,1] \rightarrow\left[\frac{1}{2}, 1\right]$ given by $g(x)=\frac{1+x}{2}$ is obviously subadditive. Finally a simple computation shows Property $\left(\star_{3}\right)$. Consequently, $I_{U}$ satisfies (MPT) with respect to $T_{\mathbf{L}}$ and $N$.

\subsection{Case When $U$ is an Idempotent Uninorm}

In this section we will deal with $R U$-implications derived from idempotent uninorms, that is, uninorms $U \equiv\langle g, e\rangle_{\text {ide }}$ with neutral element $e \in[0,1]$ and such that $g(0)=1$. Recall that for this kind of uninorms, the corresponding RUimplications have the following structure.

Proposition 6 ([20]). Let $U \equiv\langle g, e\rangle_{\text {ide }}$ be an idempotent uninorm with neutral element $e \in] 0,1\left[\right.$ and such that $g(0)=1$. Then $I_{U}$ is given by

$$
I_{U}(x, y)= \begin{cases}\max (g(x), y) & \text { if } x \leq y, \\ \min (g(x), y) & \text { if } x>y\end{cases}
$$

From results in [15], for an idempotent uninorm $U \equiv\langle g, e\rangle_{\text {ide }}$ with $g(0)=1$, as $T_{U}=\min , I_{U}$ satisfies (MP) with respect to any t-norm. Therefore, we can write the following result.

Proposition 7. Let $U \equiv\langle g, e\rangle_{\text {ide }}$ be an idempotent uninorm with neutral element $e \in] 0,1[$ and such that $g(0)=1, T$ a t-norm and $N$ a fuzzy negation. Then $I_{U}$ satisfies (MPT) with respect to $T$ and $N$ if and only if $I_{U}$ satisfies (MT) with respect to $T$ and $N$.

Thus, in the rest of the section, all the conditions in the results will be related to the fulfillment of (MT) (that was studied in [14]), which will imply the fulfillment of (MPT). Now we will distinguish two cases depending on the value of $g(1)$. We will start with the case $g(1)>0$.

Proposition 8. Let $U \equiv\langle g, e\rangle_{i d e}$ with $g(0)=1$ and $g(1)>0$ and $I_{U}$ its residual implication. Let $T$ be a t-norm and $N$ a continuous fuzzy negation. If $I_{U}$ satisfies the (MPT) property with respect to $T$ and $N$, then the following statements are true: 
(i) $T(N(y), y)=0$ for all $y \leq g(1)$.

(ii) If $T$ is a continuous t-norm then $T$ must be nilpotent with normalized additive generator $t:[0,1] \rightarrow[0,1]$ and associated negation $N_{T}$, which is given by $N_{T}(x)=t^{-1}(1-t(x))$, such that $N(y) \leq N_{T}(y)$ for all $y \leq g(1)$.

Although this result provides only necessary conditions on $T$, the following example gives infinite cases of residual implications $I_{U}$ from $U$ an idempotent uninorm such that satisfy (MPT) with respect a t-norm $T$ and a strong negation $N$.

Example 7. Consider $U \equiv\langle g, e\rangle_{\text {ide }}$ any idempotent uninorm, $T=T_{\mathbf{L}}$ the tukasiewicz t-norm and $N=N_{c}$ the classical negation. Take $x, y \in[0,1]$ such that $y<x$, then we have:

$$
T_{\mathbf{L}}\left(N_{c}(y), \min (g(x), y)\right)=\max (0, \min (g(x), y)-y)=0,
$$

and, by Remark 1, (MT) is satisfied and by Proposition 7, $I_{U}$ satisfies (MPT) with respect to $T_{\mathbf{L}}$ and $N_{c}$. If $g \neq N, I_{U}$ satisfies (MPT) with respect to $T_{\mathbf{L}}$ and $N_{c}$ but $I_{U}$ does not have $(\mathbf{C P})$ with respect to $N_{c}$.

When $g(1)=0$ we have a first result which can be applied for any fuzzy negation $N$.

Theorem 5. Let $U \equiv\langle g, e\rangle_{\text {ide }}$ be an idempotent uninorm with neutral element $e \in] 0,1\left[\right.$ and $g(0)=1, g(1)=0$ and $I_{U}$ its residual implication. Let $T$ be a $t$ norm and $N$ a continuous fuzzy negation. Then $I_{U}$ satisfies (MPT) with respect to $T$ and $N$ if and only if

$$
\min (T(N(y), y), T(N(y), g(x))) \leq N(x) \text { for all } y<x .
$$

Example 8. Let us consider $U \equiv\left\langle N_{c}, \frac{1}{2}\right\rangle_{\text {ide }}$ an idempotent uninorm, $T=T_{\mathbf{L}}$ and $N=N_{c}$. Similarly to the previous Example 7 we have

$$
\min \left(T_{\mathbf{L}}\left(N_{c}(y), y\right), T_{\mathbf{L}}\left(N_{c}(y), g(x)\right)\right)=0
$$

and then $I_{U}$ satisfies (MPT) with respect to $T_{\mathbf{L}}$ and $N_{c}$.

When $N$ is strict, the following result provides an easier condition in order to verify the fulfillment of (MPT).

Proposition 9. Let $T$ be a t-norm, $N$ a strict fuzzy negation, and $U \equiv\langle g, e\rangle_{\text {ide }}$ be an idempotent uninorm with neutral element $e \in] 0,1[$ with $g(0)=1, g(1)=0$ and $I_{U}$ its residual implication. Then $I_{U}$ satisfies (MPT) with respect to $T$ and $N$ if and only if $g(x) \leq N(x)$ for all $x \geq e$.

Example 9. Let us consider $U \equiv\left\langle g, \frac{1}{4}\right\rangle_{\text {ide }}$ an idempotent uninorm where

$$
g(x)= \begin{cases}1-3 x & \text { if } x \leq \frac{1}{3} \\ 0 & \text { otherwise }\end{cases}
$$

$T=T_{\mathbf{P}}$ and $N=N_{c}$. It is straightforward to prove that $g(x) \leq N(x)$ for all $x \geq \frac{1}{4}$. Then, we are under the conditions of the previous result. Thus, $I_{U}$ satisfies (MPT) with respect to $T$ and $N$. 


\subsection{Case When $U$ is a Representable Uninorm}

In this section we will deal with $R U$-implications derived from representable uninorms, that is, from uninorms $U \equiv\langle h, e\rangle_{\text {rep }}$ with neutral element $\left.e \in\right] 0,1[$. Let us recall in this case the expression of the residual implication derived from $U$.

Proposition 10 (Theorem 5.4.10 in [3]). Let $U \equiv\langle h, e\rangle_{\text {rep }}$ be a representable uninorm with neutral element $e \in] 0,1\left[\right.$. Then $I_{U}$ is given by

$$
I_{U}(x, y)= \begin{cases}1 & \text { if }(x, y) \in\{(0,0),(1,1)\} \\ h^{-1}(h(y)-h(x)) & \text { otherwise. }\end{cases}
$$

For this kind of uninorms we will consider only continuous t-norms which are not an ordinal sum, namely, the minimum t-norm and continuous Archimedean t-norms.

Proposition 11. Let $U \equiv\langle h, e\rangle_{\text {rep }}$ be a representable uninorm with neutral element $e \in] 0,1\left[\right.$ and $I_{U}$ its residual implication. Let $T$ be a continuous nonordinal sum t-norm and $N$ a continuous fuzzy negation. Then, it holds that:

- If $I_{U}$ satisfies (MPT) with $T$ and $N$, then $T$ is continuous Archimedean with additive generator $t:[0,1] \rightarrow[0,+\infty]$, up to a multiplicative constant.

Thus, in this case, the following statements are true:

(i) $I_{U}$ satisfies (MPT) with respect to $T$ and $N$ if and only if Property $\left(\star_{1}\right)$ is fulfilled and the following property holds:

$(\bullet 1)$ For all $y \leq x$,

$$
h^{-1}(h(y)-h(x)) \leq t^{-1}(t(N(x))-t(N(y))) .
$$

(ii) If $T$ is nilpotent and $N=N_{T}, I_{U}$ satisfies (MPT) with respect to $T$ and $N$ if and only if the following property holds:

$\left(\bullet_{2}\right)$ Function $\phi:[0,1] \rightarrow[-\infty,+\infty]$ given by $\phi(x)=h\left(t^{-1}(x)\right)$ for all $x \in$ $[0,1]$ is subadditive.

Example 10. Let us consider $T=T_{\mathbf{L}}$ and $N=N_{c}$. Let $U$ be the representable uninorm given by

$$
U(x, y)= \begin{cases}0 & \text { if }(x, y) \in\{(0,0),(1,1)\} \\ \frac{x y}{x y+(1-x)(1-y)} & \text { otherwise }\end{cases}
$$

which has $e=\frac{1}{2}$ as neutral element and additive generator $h(x)=\ln \left(\frac{x}{1-x}\right)$. In this case, $\phi(x)=h\left(t^{-1}(x)\right)=\ln \left(\frac{1-x}{x}\right)$ which is clearly subadditive. By applying Case (ii) of the previous proposition, we conclude that $I_{U}$ satisfies (MPT) with respect to $T$ and $N$. 


\section{Conclusions and Future Work}

In this paper, we have studied the fulfillment of the so-called Modus Ponens Tollens property (MPT) by the family of $R U$-implications, i.e., we have analyzed which $R U$-implications satisfy at the same time the Modus Ponens and the Modus Tollens properties with respect to a t-norm $T$ and a negation $N$. From this study, many solutions are available. On the one side, all $R U$-implications which satisfy the Modus Ponens property with respect to a t-norm $T$ and the contrapositive symmetry with respect to a strong negation $N$ are solutions of (MPT). On the other side, when $N$ is not strong or the contrapositive symmetry is not satisfied, other solutions exist within $R U$-implications derived from uninorms in $\mathcal{U}_{\text {min }}$, representable uninorms and idempotent uninorms. For most of these families, necessary and sufficient conditions are presented and in some cases, it is shown that the fulfillment of the Modus Tollens property implies the fulfillment of the Modus Ponens property.

As future work, we want to complete the results presented in this paper by considering also continuous ordinal sum t-norms as $T$ in some of the results presented in Sect. 4 and to deepen the study in the particular case of idempotent uninorms with $g(0)=1$ and $g(1)>0$.

Acknowledgments. This paper has been supported by the Spanish Grant TIN201675404-P AEI/FEDER, UE.

\section{References}

1. Aguiló, I., Suñer, J., Torrens, J.: A characterization of residual implications derived from left-continuous uninorms. Inf. Sci. 180(20), 3992-4005 (2010)

2. Alsina, C., Trillas, E.: When $(S, N)$-implications are $\left(T, T_{1}\right)$-conditional functions? Fuzzy Sets Syst. 134, 305-310 (2003)

3. Baczyński, M., Jayaram, B.: Fuzzy Implications. Studies in Fuzziness and Soft Computing, vol. 231. Springer, Heidelberg (2008). https://doi.org/10.1007/978-3540-69082-5

4. Baczynski, M., Jayaram, B., Massanet, S., Torrens, J.: Fuzzy implications: past, present, and future. In: Kacprzyk, J., Pedrycz, W. (eds.) Springer Handbook of Computational Intelligence, pp. 183-202. Springer, Heidelberg (2015). https://doi. org/10.1007/978-3-662-43505-2_12

5. Bustince, H., Burillo, P., Soria, F.: Automorphisms, negations and implication operators. Fuzzy Sets Syst. 134, 209-229 (2003)

6. De Baets, B.: Idempotent uninorms. Eur. J. Oper. Res. 118, 631-642 (1999)

7. De Baets, B., Fodor, J.C.: Residual operators of uninorms. Soft. Comput. 3, 89-100 (1999)

8. Dimuro, G.P., Bedregal, B.R.C., Fernández, J., Sesma-Sara, M., Pintor, J.M., Bustince, H.: The law of O-conditionality for fuzzy implications constructed from overlap and grouping functions. Int. J. Approx. Reason. 105, 27-48 (2019)

9. Fodor, J.C., Yager, R.R., Rybalov, A.: Structure of uninorms. Int. J. Uncertainty Fuzziness Knowl.-Based Syst. 5, 411-427 (1997)

10. Hu, S., Li, Z.: The structure of continuous uninorms. Fuzzy Sets Syst. 124, 43-52 (2001) 
11. Klement, E.P., Mesiar, R., Pap, E.: Triangular Norms. Kluwer Academic Publishers, Dordrecht (2000)

12. Martín, J., Mayor, G., Torrens, J.: On locally internal monotonic operators. Fuzzy Sets Syst. 137, 27-42 (2003)

13. Mas, M., Massanet, S., Ruiz-Aguilera, D., Torrens, J.: A survey on the existing classes of uninorms. J. Intell. Fuzzy Syst. 29(3), 1021-1037 (2015)

14. Mas, M., Monreal, J., Monserrat, M., Riera, J.V., Torrens Sastre, J.: Modus Tollens on fuzzy implication functions derived from uninorms. In: Calvo Sánchez, T., Torrens Sastre, J. (eds.) Fuzzy Logic and Information Fusion. SFSC, vol. 339, pp. 49-64. Springer, Cham (2016). https://doi.org/10.1007/978-3-319-30421-2_5

15. Mas, M., Monserrat, M., Ruiz-Aguilera, D., Torrens, J.: RU and (U, N)implications satisfying Modus Ponens. Int. J. Approx. Reason. 73, 123-137 (2016)

16. Mas, M., Monserrat, M., Torrens, J.: Modus Ponens and Modus Tollens in discrete implications. Int. J. Approx. Reason. 49, 422-435 (2008)

17. Mas, M., Monserrat, M., Torrens, J.: A characterization of $(U, N), R U, Q L$ and $D$ implications derived from uninorms satisfying the law of importation. Fuzzy Sets Syst. 161, 1369-1387 (2010)

18. Mas, M., Monserrat, M., Torrens, J., Trillas, E.: A survey on fuzzy implication functions. IEEE Trans. Fuzzy Syst. 15(6), 1107-1121 (2007)

19. Mas, M., Ruiz-Aguilera, D., Torrens, J.: Uninorm based residual implications satisfying the Modus Ponens property with respect to a uninorm. Fuzzy Sets Syst. 359, 22-41 (2019)

20. Ruiz, D., Torrens, J.: Residual implications and co-implications from idempotent uninorms. Kybernetika 40, 21-38 (2004)

21. Ruiz-Aguilera, D., Torrens, J.: $S$ - and $R$-implications from uninorms continuous in ]0, $1\left[^{2}\right.$ and their distributivity over uninorms. Fuzzy Sets Syst. 160, 832-852 (2009)

22. Ruiz-Aguilera, D., Torrens, J., De Baets, B., Fodor, J.: Some remarks on the characterization of idempotent uninorms. In: Hüllermeier, E., Kruse, R., Hoffmann, F. (eds.) IPMU 2010. LNCS (LNAI), vol. 6178, pp. 425-434. Springer, Heidelberg (2010). https://doi.org/10.1007/978-3-642-14049-5_44

23. Trillas, E., Alsina, C., Pradera, A.: On MPT-implication functions for fuzzy logic. Revista de la Real Academia de Ciencias. Serie A. Matemáticas (RACSAM) 98(1), 259-271 (2004)

24. Trillas, E., Alsina, C., Renedo, E., Pradera, A.: On contra-symmetry and MPTconditionality in fuzzy logic. Int. J. Intell. Syst. 20, 313-326 (2005)

25. Trillas, E., Valverde, L.: On Modus Ponens in fuzzy logic. In: Proceedings of the 15th International Symposium on Multiple-Valued Logic, Kingston, Canada, pp. 294-301 (1985) 